\title{
Classic hybrid arch debranching (type I hybrid arch repair) without circulatory arrest
}

\author{
G. Chad Hughes \\ Division of Cardiovascular and Thoracic Surgery, Department of Surgery, Duke University Medical Center, Durham, North Carolina, USA \\ Correspondence to: G. Chad Hughes, MD. Box 3051 DUMC, Durham, NC 27710, USA. Email: gchad.hughes@duke.edu.
}

Submitted Mar 27, 2018. Accepted for publication May 03, 2018.

doi: $10.21037 /$ acs.2018.05.06

View this article at: http://dx.doi.org/10.21037/acs.2018.05.06

\section{Background}

Like most surgical procedures, patient selection is critical if one is to obtain good results with the classic hybrid arch debranching procedure [type I hybrid arch repair (HAR)]. This procedure is now mostly utilized at our institution for patients status post prior type A dissection repair with aortic arch and descending thoracic aortic dilation, but who are not felt to be a candidates for more extensive type II or III HAR requiring total arch replacement. As detailed below, we have become very selective with our use of this procedure for the patient with native zone 0 due to the risk of retrograde type A dissection and will only perform a type I HAR if the native ascending aorta measures $<4 \mathrm{~cm}$ in diameter. The following vignette is a typical case for type I HAR at our institution.

\section{Clinical vignette}

The patient is an 84-year-old male post prior supracoronary ascending aorta and hemi-arch replacement for acute type A dissection at another institution 8 months prior to referral to the Duke Center for Aortic Disease. His comorbidities include hypertension, hyperlipidemia, and paroxysmal atrial fibrillation. Over the past month or so, he has developed new onset hoarseness and cough and further workup by his primary care physician with computed tomography angiography (CTA) discovered a new 8.4-cm aneurysm of his distal aortic arch and proximal descending thoracic aorta secondary to residual dissection (Figure 1). Transthoracic echocardiography demonstrates an ejection fraction of $>55 \%$ with trivial aortic insufficiency. Pulmonary function testing reveals no significant obstructive lung disease with mildly reduced diffusing capacity of the lung for carbon monoxide (DLCO). Centerline reconstruction CTA images (Figure $2 A, B, C$ ) demonstrate a long $(>5 \mathrm{~cm}$ ) length of Dacron aorta above the proposed inflow site for an arch debranching graft that would serve as adequate proximal landing zone (PLZ) for a type I HAR. Mean diameter of the ascending Dacron graft PLZ measures $32 \mathrm{~mm}$ (Figure 2D) and of the true lumen distal landing zone (DLZ) above the celiac axis $21 \mathrm{~mm}$ (Figure 2E). Given his age and frailty, the patient was not felt to be a good candidate for a more extensive type II or III HAR involving total arch replacement with hypothermic circulatory arrest. He was deemed a screen fail by the screening committee for an investigational zone 0 device trial ongoing at our institution, and therefore a type I HAR was recommended.

\section{Surgical techniques}

The operative plan was for first-stage arch debranching to include re-implantation of all three arch vessels into a trifurcated Dacron graft to be anastomosed to the most proximal extent of the ascending aortic Dacron graft via redo sternotomy on cardiopulmonary bypass (CPB) followed by $2^{\text {nd }}$-stage completion thoracic endovascular aortic repair (TEVAR) during the same hospital stay. The decision for implantation of the left subclavian artery (LSCA) is based upon the ease of reaching this vessel from the front via sternotomy as detailed below, but in this case the LSCA arose in close proximity to the left common carotid artery (LCCA) and was felt to be re-implantable from the front (Figure 2A). Given his hoarseness secondary to left recurrent laryngeal nerve palsy from stretch injury by the large aneurysm, direct microlaryngoscopy with vocal cord injection with cymetra by the otolaryngology service 

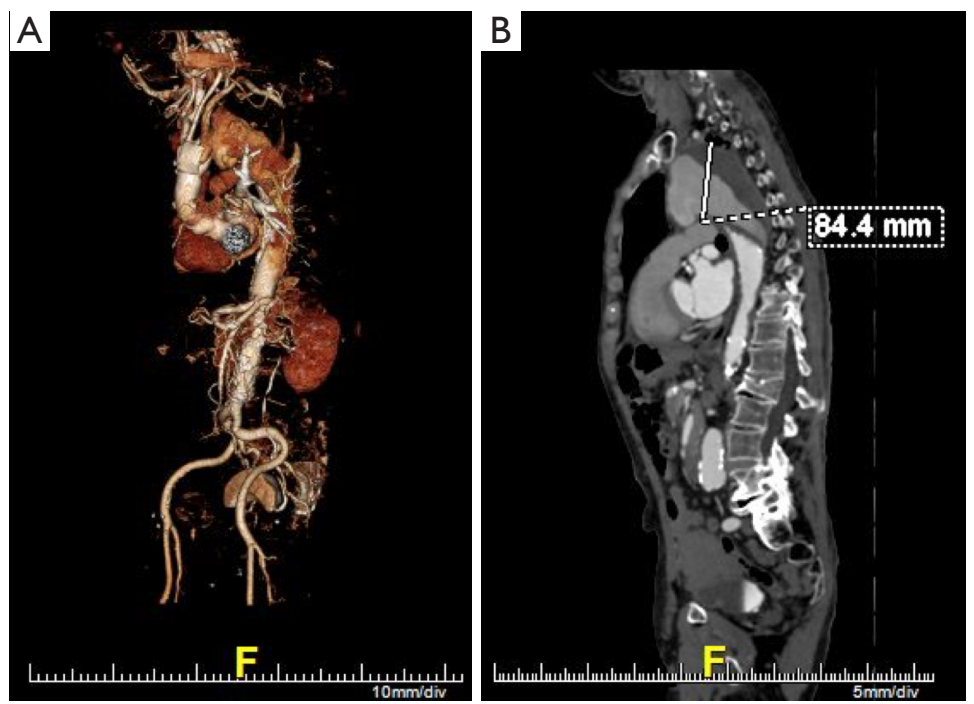

Figure 1 Preoperative 3D (A) and sagittal reconstructed (B) CTA images demonstrating $>8 \mathrm{~cm}$ diameter aneurysm of the distal aortic arch and proximal descending thoracic aorta secondary to chronic residual dissection after prior hemi-arch repair of acute type A dissection. CTA, computed tomography angiography.

at the beginning of the case was planned as well, as we have found postoperative pulmonary toilet and swallowing function to be improved when concurrent vocal cord injection is performed in this scenario. Details of the type I HAR procedure are given below.

\section{Preparation}

The patient is positioned supine on the operating room table with the arms tucked at the sides. A small bump or roll is placed beneath the patient's shoulder blades to assist with neck extension so as to facilitate exposure of the supraaortic arch vessels. Bilateral radial arterial lines and a central venous line are placed by the cardiac anesthesia team. Electroencephalography (EEG) leads are placed by the neurophysiologic monitoring service.

\section{Exposition}

The operation begins with right axillary cannulation using an 8 -mm side graft technique, as we have evolved to favor performing all of these procedures using beating heart CPB. After axillary cannulation is complete, the debranching portion of the operation is done via median sternotomy with the incision extended cephalad along either the right or left sternocleidomastoid muscle (SCM) to allow dissection and mobilization of the arch vessels in the base of the neck. If only the innominate and LCCA are to be debranched, then the incision typically is extended along the right SCM (Figure 3A), whereas, as in the current case, the incision is extended along the left SCM if the LSCA will be debranched as well (Figure 3B). In the redo median sternotomy setting, again as in the current case, a femoral venous line is placed on the right to facilitate percutaneous femoral venous cannulation should this be necessary due to dense mediastinal adhesions.

\section{Operation}

The heart is suspended in a pericardial cradle. We prefer to mobilize the arch vessels prior to full heparinization and institution of $\mathrm{CPB}$. Once the arch vessels have been mobilized, attention is turned to the ascending aorta. As noted above, a majority of these cases in our current practice are patients with Dacron proximal aorta status post prior type A dissection repair, and the ascending/ hemi-arch graft will need to be mobilized down to as close to the proximal anastomosis at the sinotubular junction as possible so as to create maximal PLZ length. This is done by incising the fibrous sheath that forms around the graft with a 15 blade and then carefully excising the fibrous peel with electrocautery such that the proximal end of the graft is mobilized enough to allow a partial clamp to be placed on the graft for performance of the proximal anastomosis 

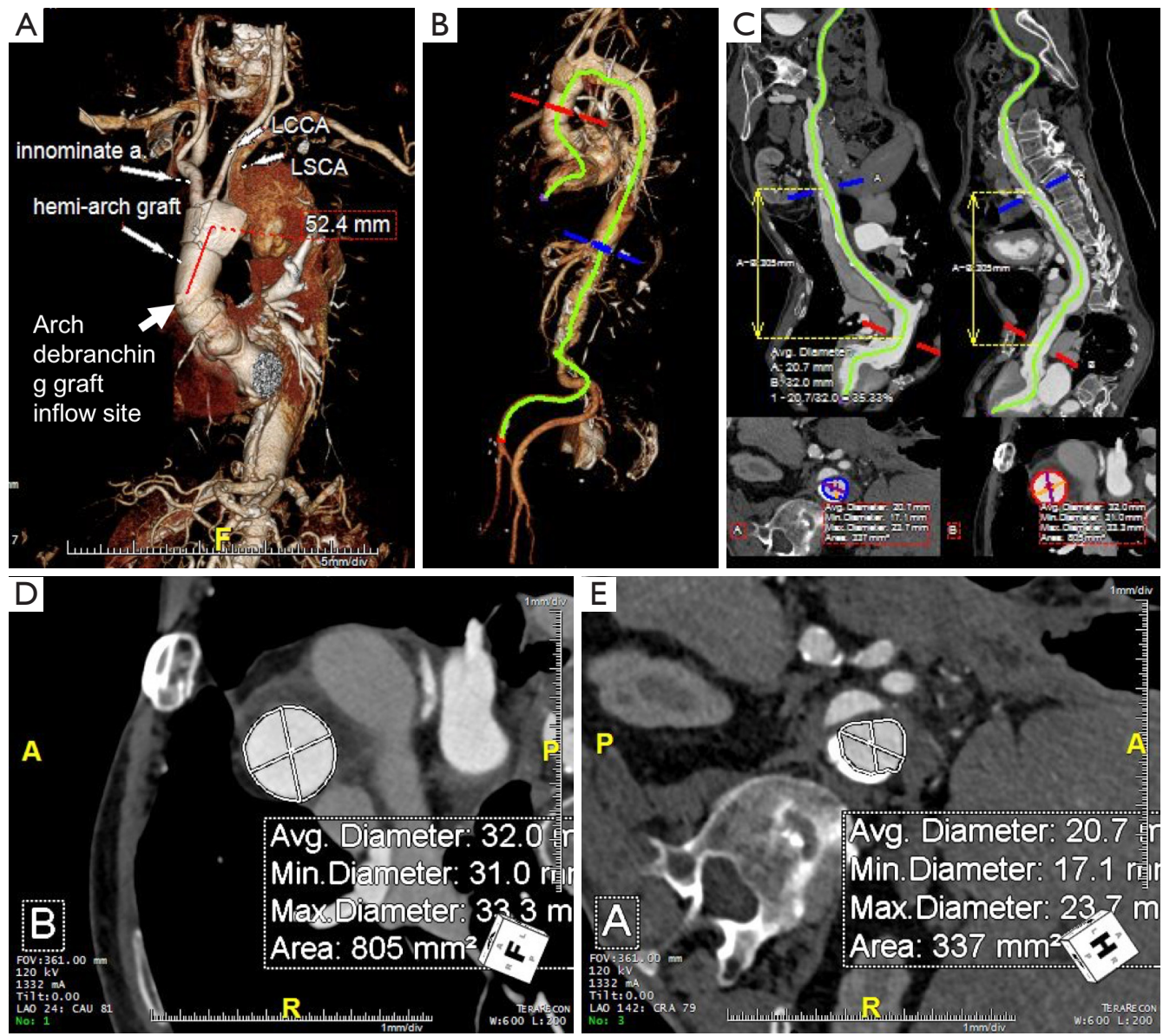

Figure 2 Centerline reconstruction CTA images (A-C) demonstrate $>5 \mathrm{~cm}$ of potential Dacron proximal landing zone above the proposed inflow site of an arch debranching graft (A). The proximal landing zone within the existing ascending aortic Dacron graft mean diameter was $32 \mathrm{~mm}(\mathrm{D})$ and the distal landing zone in the true lumen at the celiac axis measured $21 \mathrm{~mm}(\mathrm{E})$. CTA, computed tomography angiography; LCCA, left common carotid artery; LSCA, left subclavian artery.
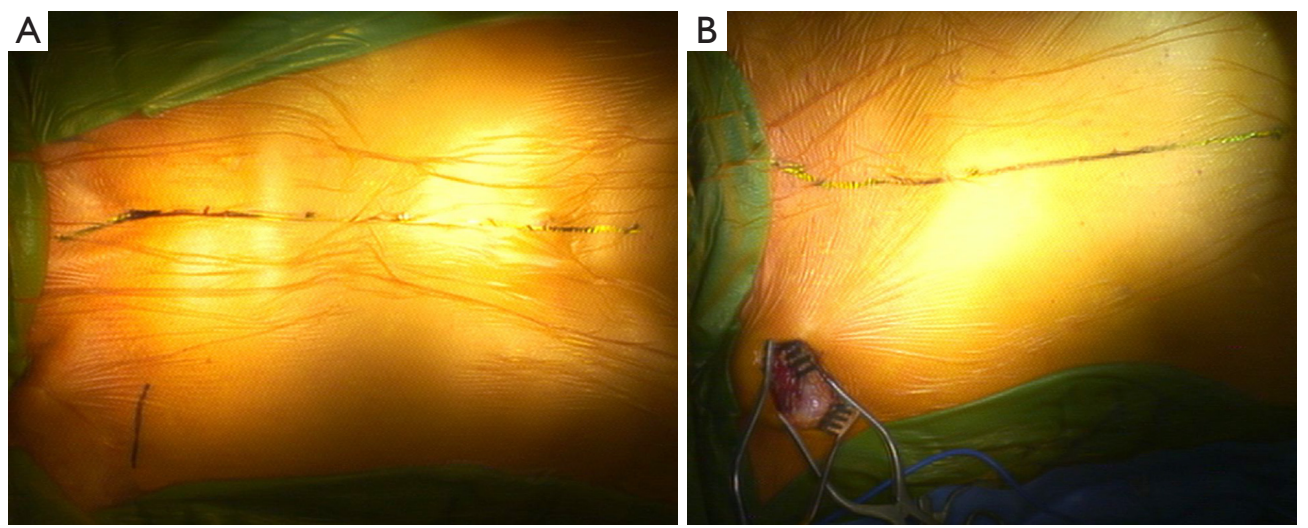

Figure 3 Intraoperative photographs demonstrating incision sites for type I HAR. If only the innominate and LCCA are to be re-implanted then the incision is typically extended cephalad along the right sternocleidomastoid muscle (A), whereas the incision is typically extended to the left if all three arch vessels are to be re-implanted from the front (B). HAR, hybrid arch repair; LCCA, left common carotid artery. 


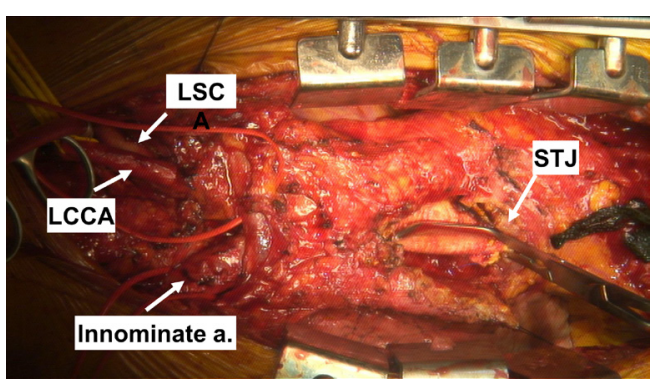

Figure 4 Intraoperative photograph from the case presented in the clinical vignette demonstrating the proximal end of the ascending aortic Dacron graft mobilized down to the sinotubular junction (STJ) to allow partial clamp placement and create maximal PLZ length. The three arch vessels have been fully mobilized and a vessel loop is seen encircling the left innominate vein. Downward tension on this vessel loop during the arch debranching distal anastomoses facilitates exposure of the arch vessels. LCCA, left common carotid artery; LSCA, left subclavian artery; PLZ, proximal landing zone.

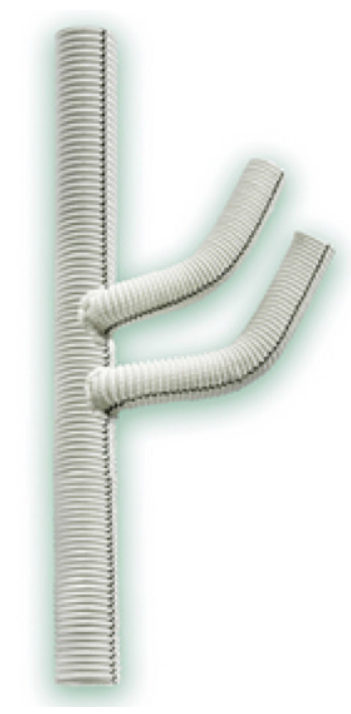

Figure 5 Photograph of commercially available trifurcated woven Dacron graft used for arch debranching. The graft is available with 14- and 12-mm main body sizes with 8 -mm limbs, and the graft size is chosen to best match the diameter of the innominate artery.

(Figure 4). This is most easily done with the patient on full $\mathrm{CPB}$ support, and therefore once the arch vessels have been mobilized, the patient is fully heparinized for CPB. In the native zone 0 setting, the ascending aorta is fully mobilized and a site chosen on the proximal ascending aorta for the inflow anastomosis of the arch debranching graft to create long segment PLZ.

In the primary sternotomy setting, the right atrial appendage is cannulated with a dual stage venous cannula. In the redo setting, the right atrium is often densely adherent to the lateral pericardium from prior venous cannulation, and therefore we have a low threshold for using percutaneous femoral venous cannulation with the femoral multistage cannula passed up into the proximal superior vena cava (SVC) under transesophageal echo guidance. The final placement of the femoral venous cannula can also be confirmed by manually palpating the cannula tip in the SVC given that the chest is open.

The patient is then placed on CPB and cooled to 34 degrees nasopharyngeal (NP) with the heart empty and beating. We use a trifurcated woven Dacron graft for the debranching procedure (Figure 5). The size of the graft is most commonly a $14-\mathrm{mm}$ main body graft with two 8 -mm distal limbs, although a 12 -mm main body graft is occasionally used if the innominate artery is small. The proximal anastomosis is then performed by anastomosing the trifurcated head vessel graft to the existing ascending Dacron graft or native ascending aorta. In either case, the anastomosis should be placed as low as possible on the aorta/ graft to create maximal PLZ length. Care should be taken to turn the CPB flow down during the application of the partial clamp, especially when clamping a native ascending aorta. Another important point is that the main body of the trifurcated graft frequently must be cut shorter so that the distal end of the graft limbs reaches the arch vessel branches properly without excessive length. This proximal anastomosis is done with running 4-0 polypropylene, and when completed the flows are again turned down and the partial clamp released. If needed, reinforcing sutures of pledgeted 4-0 polypropylene may be placed to address any areas of bleeding around the anastomosis, again turning the pump flows down as needed to facilitate suture placement and during tying of the suture knots (Figure 6). Radiographic markers are then placed around the proximal anastomosis to facilitate its identification under fluoroscopy and to mark the most proximal extent of the PLZ so as to avoid inadvertently covering the anastomosis with the most proximal endograft at the $2^{\text {nd }}$-stage TEVAR procedure (Figure 7).

Attention is then turned to the arch vessels. If all three arch vessels are to be re-implanted, the LSCA is debranched first. We first perform a test clamp of each arch vessel for 2 minutes prior to debranching to confirm no EEG changes suggestive of ischemia occur; in the rare instance 


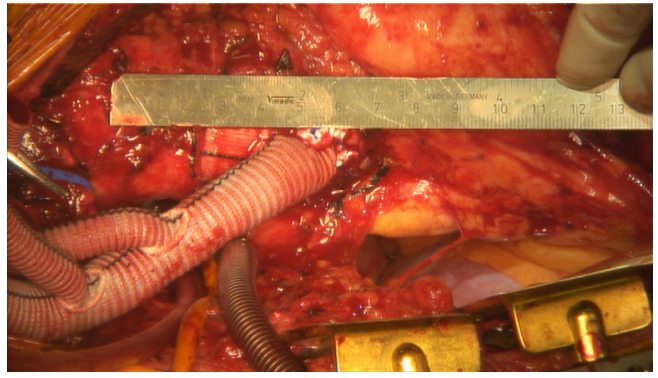

Figure 6 Intraoperative photograph from another patient undergoing type I HAR after prior type A dissection repair demonstrating the completed proximal anastomosis and reinforcing sutures of pledgeted 4-0 Prolene. A ruler demonstrates the creation of nearly $5 \mathrm{~cm}$ of Dacron PLZ in this case. HAR, hybrid arch repair; PLZ, proximal landing zone.

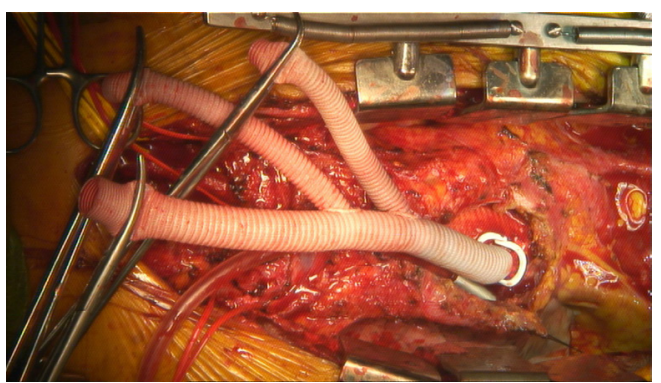

Figure 7 Intraoperative photograph from the case presented in the clinical vignette demonstrating completed proximal anastomosis and radiographic markers placed around the debranching graft origin to facilitate its identification under fluoroscopy.

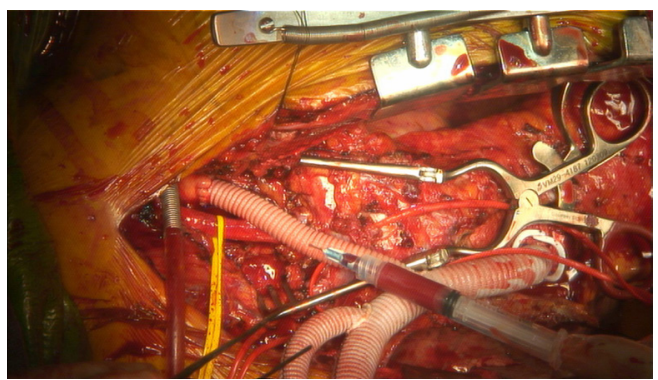

Figure 8 Intraoperative photograph from the case presented in the clinical vignette demonstrating completed distal anastomosis to the LSCA with the graft limb being carefully de-aired using a 25 -gauge needle. The graft limb is also de-aired extensively prior to re-establishing antegrade flow via temporary release of the distal clamp on the LSCA prior to tying the suture knots. The importance of careful graft de-airing cannot be over emphasized to avoid cerebral embolization. LSCA, left subclavian artery.
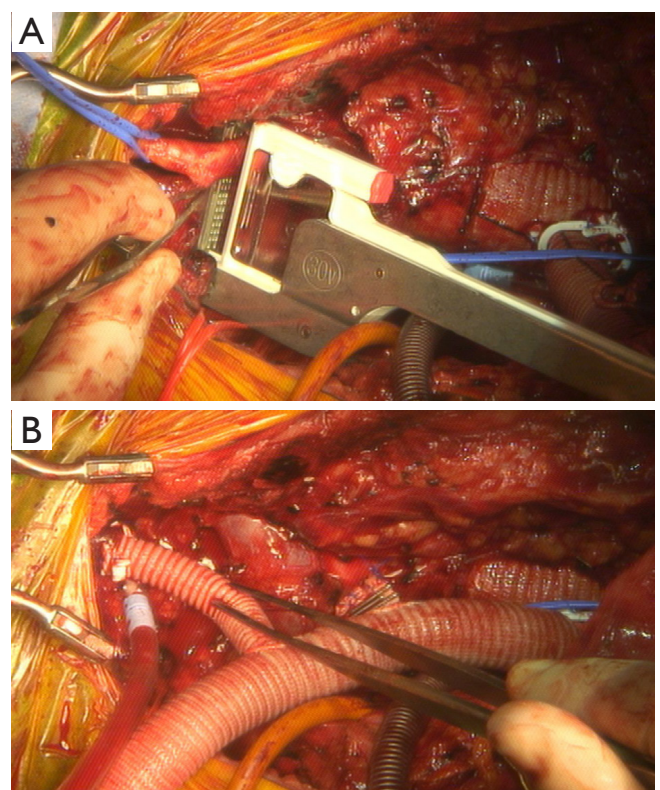

Figure 9 Intraoperative photograph from the same patient as shown in Figure 7 and in whom only the LCCA and innominate artery were re-implanted. The photo in (A) shows the proximal LCCA being stapled with a vascular stapler prior to applying a clamp on the distal LCCA and dividing it above the staple line. The completed anastomosis to the LCCA is shown in (B). LCCA, left common carotid artery.

this is noted, the blood pressure may be augmented and the patient further cooled towards 28 degrees on CPB which allows a longer safe duration of vessel clamping. As each anastomosis takes no more than 5-8 minutes to perform, this strategy works well and we have not had issues with cerebral ischemia induced by debranching. The proximal LSCA is then stapled with a vascular stapler proximally, clamped distally, and divided. The distal $8-\mathrm{mm}$ limb of the debranching graft is cut to length and anastomosed end-to-end to the LSCA with running 5-0 polypropylene suture. The graft is carefully and meticulously de-aired and antegrade flow then re-established to the LSCA (Figure 8). This process is then repeated for the LCCA (Figure 9).

Of note, in cases where the LSCA arises too far distal on the arch to re-implanted from the front via the sternotomy, we perform LCCA-LSCA bypass first at the beginning of the case. By performing this initial bypass, the left carotid distribution is fed via the LSCA during the period of LCCA proximal clamp, and there is no need for a test clamp in this situation (Figure 10).

At this point, the heart is allowed to fill, the lungs 


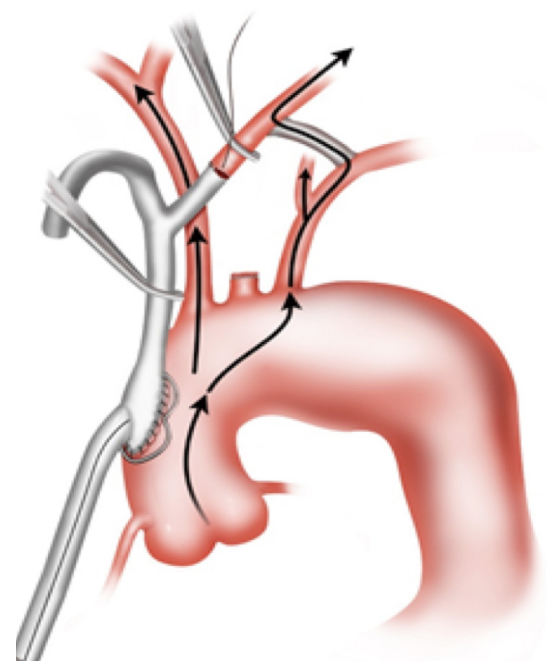

Figure 10 Drawing of a native zone 0 type I HAR in which LCCA to LSCA bypass has been performed prior to arch debranching. The LCCA-LSCA bypass will provide flow to the LCCA distribution during LCCA debranching and therefore a test clamp is not needed in this setting as the LCCA distribution will not be ischemic. HAR, hybrid arch repair; LSCA, left subclavian artery; LCCA, left common carotid artery.

are ventilated, and the patient weaned from CPB. The innominate artery is then clamped and low flow antegrade cerebral perfusion (ACP) begun via the axillary graft to a right radial arterial line pressure of $70 \mathrm{mmHg}$, such that the innominate distribution is not ischemic during the period of innominate debranching. As such, a test clamp is not required (Figure 11). The innominate is then debranched in a similar manner to the other arch vessels (Figure 12). The patient is then decannulated, protamine administered, and hemostasis obtained. The chest is closed in the usual manner. We place a 24-Fr Blake drain in the chest adjacent to the debranching graft that is left in place for 72 hours and until the drainage is $<150 \mathrm{cc} / 24$ hours to prevent the formation of peri-graft seroma.

The patient remains in the hospital until the $2^{\text {nd }}$-stage TEVAR completion procedure. This is performed in a hybrid operating room, typically $3-5$ days after the firststage debranching procedure. The procedure is done in standard fashion with the direction of endograft deployment (proximal to distal or vice versa) depending on patient
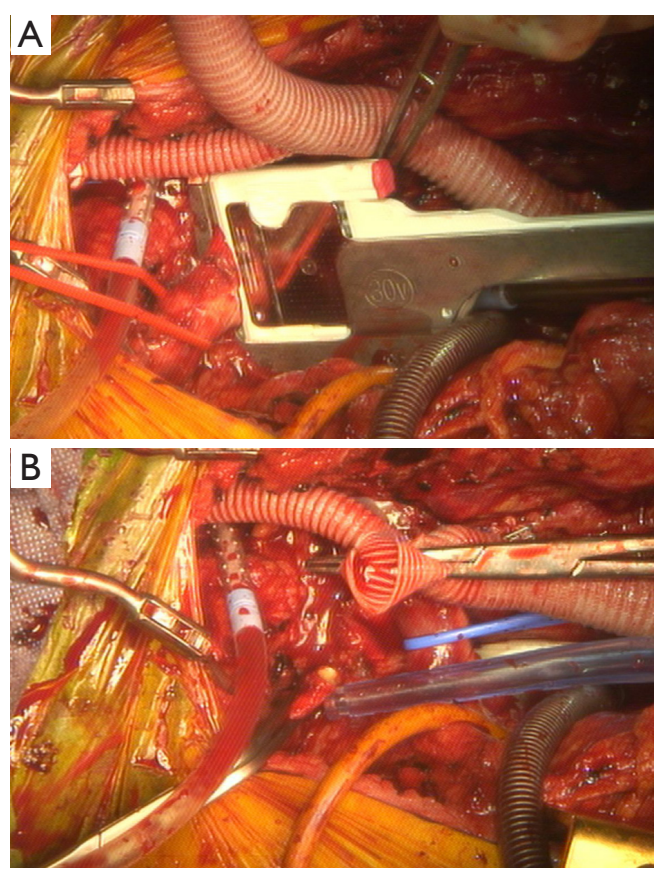

Figure 11 Intraoperative photograph from the same patient as shown in Figures 6,9 demonstrating the innominate artery being stapled proximally at its origin from the aorta with a vascular stapler (A). The innominate artery is then clamped distally and divided above the staple line (B). The innominate distribution is perfused via the right axillary side graft during this period to a target right radial arterial line pressure of $70 \mathrm{mmHg}$ such that the innominate distribution is not ischemic during the period of debranching. The patient has otherwise been weaned from CPB at this point with the heart ejecting and the lungs ventilated such that the left brain and body are perfused via the native circulation. $\mathrm{CPB}$, cardiopulmonary bypass.

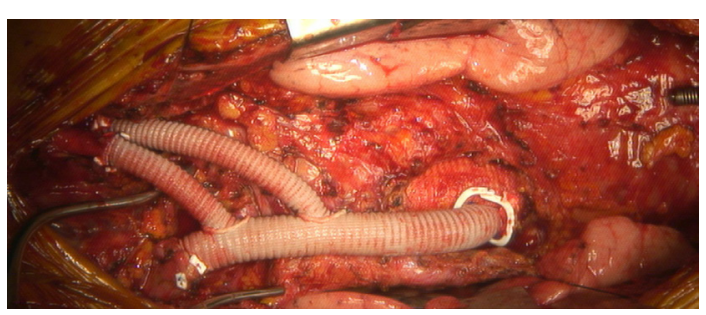

Figure 12 Intraoperative photograph from the case presented in the clinical vignette demonstrating completed stage I arch debranching procedure. 
anatomy. The radiographic markers placed around the debranching graft at the first operation are used to guide the most proximal extent of endograft placement.

\section{Completion}

Patients typically spend one night in the cardiothoracic intensive care unit (CT-ICU) following the first-stage debranching procedure. Our protocol is for 4 hours of CTICU monitoring following the $2^{\text {nd }}$-stage TEVAR procedure, at which time the patient is transferred to the stepdown unit for the remainder of their hospital stay. Most patients are discharged 2 days following $2^{\text {nd }}$-stage TEVAR.

\section{Comments}

\section{Clinical results}

Following commercial availability of thoracic endografts, HAR was developed as an alternative to conventional open repair with the potential advantages of reduced perioperative morbidity and mortality, as well as allowing repair in some higher risk patients not considered candidates for open surgery (1). Early reports with these techniques, including data from our own institution (2), appeared promising, although with increasing experience the limitations of this so-called type I HAR involving arch debranching with native ascending aorta (zone 0) landing zone became apparent (3). However, with our current more restrictive criteria for patient selection including at least 4-5 cm of Dacron PLZ (4) in the patient status post prior proximal aortic repair and ascending aortic diameter $<4 \mathrm{~cm}$ in the patient with native zone 0 (5), as well as the use of $\mathrm{CPB}$ in all cases to avoid side biting clamp placement on a pressurized native aorta with use of mild hypothermia to prolong the safe duration of cerebral ischemia, results have been excellent with avoidance of retrograde dissection.

\section{Advantages}

In patients with appropriate anatomy, the type I HAR done using beating heart CPB with mild hypothermia is an excellent option for the treatment of aortic arch \pm concomitant descending thoracic aortic pathology. The procedure avoids aortic cross-clamp and therefore does not involve cardioplegic arrest of the heart, which makes it well tolerated even in patients with reduced cardiac function. Further, the use of mild hypothermia adds additional cerebral protection during the period of arch debranching. By dividing the repair into two stages, the physiologic insult is lessened, thereby making it well tolerated even in patients with significant comorbidities such as pulmonary and renal disease. We also prefer staged repair given the competing postoperative management strategies with regard to blood pressure following open proximal versus endovascular distal aortic repair (3). Specifically, lower mean arterial pressures are preferred in the early postoperative period to mitigate bleeding risk after the debranching procedure, especially in the redo setting, whereas bleeding is generally not an issue after endovascular distal aortic repair and higher mean arterial pressures are preferred for spinal cord protection. Similar to our protocol for hybrid thoracoabdominal aortic repair (6), staged repair also reduces the nephrotoxic insult of prolonged surgery followed by contrast administration, reduces blood loss by limiting the period of heparinization, and allows for patient recovery and medical optimization before the $2^{\text {nd }}$-stage procedure.

\section{Caveats}

As noted above, with increasing experience the limitations of the type I HAR became apparent (3), including the devastating complication of retrograde type A dissection, which our group was the first to link to this procedure in the setting of even modest $(>4.0 \mathrm{~cm})$ ascending aortic dilation (5). As a result of these limitations, over the past decade our group has shifted away from the native zone 0 HAR (7) towards increasing use of a two-stage approach involving first stage proximal aortic replacement including ascending aorta ( \pm valve, \pm root) and total arch replacement with (type III HAR) or without (type II HAR) creation of an elephant trunk followed by $2^{\text {nd }}$-stage TEVAR with PLZ within the Dacron replaced proximal aorta (4). However, there remain patients unsuitable for these total arch-based procedures, and the type I HAR remains a good option in this setting with the major caveat being the patient must have suitable PLZ anatomy.

\section{Acknowledgements}

None.

\section{Footnote}

Conflicts of Interest: The author has no conflicts of interest to declare. 


\section{References}

1. Hughes GC, Sulzer CF, McCann RL, et al. Endovascular approaches to complex thoracic aortic disease. Semin Cardiothorac Vasc Anesth 2008;12:298-319.

2. Hughes GC, Daneshmand MA, Balsara KR, et al. "Hybrid" repair of aneurysms of the transverse aortic arch: midterm results. Ann Thorac Surg 2009;88:1882-7.

3. Andersen ND, Williams JB, Hanna JM, et al. Results with an algorithmic approach to hybrid repair of the aortic arch. J Vasc Surg 2013;57:655-67.

4. Ganapathi AM, Andersen ND, Hanna JM, et al. Comparison of attachment site endoleak rates in

Cite this article as: Hughes GC. Classic hybrid arch debranching (type I hybrid arch repair) without circulatory arrest. Ann Cardiothorac Surg 2018;7(3):443-450. doi: 10.21037/acs.2018.05.06
Dacron versus native aorta landing zones after thoracic endovascular aortic repair. J Vasc Surg 2014;59:921-9.

5. Williams JB, Andersen ND, Bhattacharya SD, et al. Retrograde ascending aortic dissection as an early complication of thoracic endovascular aortic repair. J Vasc Surg 2012;55:1255-62.

6. Hughes GC, Barfield ME, Shah AA, et al. Staged total abdominal debranching and thoracic endovascular aortic repair for thoracoabdominal aneurysm. J Vasc Surg 2012;56:621-9.

7. Benrashid E, Wang H, Keenan JE, et al. Evolving practice pattern changes and outcomes in the era of hybrid aortic arch repair. J Vasc Surg 2016;63:323-31. 